\title{
ON DIFFERENTIABILITY AT THE BOUNDARY IN CONFORMAL MAPPING ${ }^{1}$
}

\author{
S. E. WARSCHAWSKI
}

Introduction. The object of this note is to present a short and simple proof of the following two theorems.

THEOREM 1. Suppose that $C$ is a closed rectifiable Jordan curve in the complex $\zeta$-plane and that $C$ has a continuously turning tangent in a neighborhood of one of its points, $\zeta_{0}$. Suppose, furthermore, that the tangent angle $\tau(s)$ as function of the arc length $s$ has a modulus of continuity $\omega(t)$ at the point $s_{0}$ which corresponds to $\zeta_{0}$, i.e.,

$$
\left|\tau(s)-\tau\left(s_{0}\right)\right| \leqq \omega\left(\left|s-s_{0}\right|\right) \quad\left(\left|s-s_{0}\right| \leqq \sigma\right),
$$

where $\omega(t)$ is a nondecreasing continuous function, $0 \leqq t \leqq \sigma$, and

$$
\int_{0}^{\sigma} \frac{\omega(t)}{t} d t<\infty .
$$

Let $\zeta=f(z)$ map the disk $|z|<1$ conformally onto the interior of $C$ and let $f\left(z_{0}\right)=\zeta_{0}$. Then

$$
\lim _{z \rightarrow z_{0}} \frac{f(z)-f\left(z_{0}\right)}{z-z_{0}}=f^{\prime}\left(z_{0}\right)
$$

exists for unrestricted approach in $|z| \leqq 1\left(z \neq z_{0}\right)$ and

$$
\lim _{z \rightarrow z_{0}} f^{\prime}(z)=f^{\prime}\left(z_{0}\right)
$$

in any Stolz angle with vertex at $z_{0}$. Furthermore, $f^{\prime}\left(z_{0}\right) \neq 0$.

THEOREM 2. If $\tau(s)$ is continuous along the entire curve $C$ and has $a$ modulus of continuity $\omega(t)$ along $C$, where $\omega(t)$ satisfies (1), then $f^{\prime}(z)$, if defined by (2) on $|z|=1$, is continuous in the closed disk $|z| \leqq 1$. Furthermore, if $m$ denotes an upper bound for $\left|f^{\prime}\left(e^{i \theta}\right)\right|$ then $\arg f^{\prime}\left(e^{i \theta}\right)$ and $\log \left|f^{\prime}\left(e^{i \theta}\right)\right|$ have the moduli of continuity

$$
\omega_{0}(\theta)=\omega(m \theta)+\theta
$$

and

$$
\omega_{0}^{*}(\theta)=M\left[\int_{0}^{\theta} \frac{\omega_{0}(t)}{t} d t+\theta \int_{\theta}^{\pi} \frac{\omega_{0}(t)}{t^{2}} d t\right]
$$

Received by the editors August 2, 1960.

${ }^{1}$ Sponsored by the Office of Naval Research under Contract Nonr-710(16) with the University of Minnesota. 
respectively, where $M$ is a constant.

Theorem 2 contains as a special case a result due to O. D. Kellogg [2] $\left(\omega(t)=K t^{\alpha}\right)$. Both theorems (except for the statements (3) and $\left(3^{\prime}\right)$ ) were obtained by the author in $[7$, p. 443] as special cases of a more general result whose proof was rather lengthy and complicated. Inasmuch as both of these theorems have found applications, a simple direct proof may be of some interest. ${ }^{2}$

1. Lemmas. We present first two lemmas.

Lemмa 1. Suppose that $C$ is a closed rectifiable Jordan curve which possesses the following property at one of its points $\zeta_{0}$ : there exists a subarc $\gamma$, which contains $\zeta_{0}$ as an interior point, and a constant $c>1$ such that for any two points $\zeta_{1}$ and $\zeta_{2}$ of $\gamma$ and the corresponding arc $\Delta s$ of $\gamma$ between them,

$$
\Delta s \leqq c\left|\zeta_{1}-\zeta_{2}\right|
$$

Suppose that $\zeta=f(z)$ maps the disk $|z|<1$ conformally onto the interior $\Omega$ of $C$, and that $f\left(z_{0}\right)=\zeta_{0}, z_{0}=e^{i \theta_{0}}$. Then there exists a constant $H$ such that for $|z| \leqq 1$

$$
\left|f(z)-f\left(z_{0}\right)\right| \leqq H\left|z-z_{0}\right|^{\alpha} \quad\left(\alpha=2 /(1+c)^{2}\right) .
$$

Furthermore, the arc length $s(\theta)$. as function of $\theta\left(\right.$ where $\left.z=e^{i \theta}\right)$ is $\operatorname{Lip}(\alpha)$ at $\theta=\theta_{0}$, i.e.,

$$
\left|s(\theta)-s\left(\theta_{0}\right)\right| \leqq K\left|\theta-\theta_{0}\right|^{\alpha} .
$$

CoROllary. If there exists a constant $c>1$ such that (4) holds uniformly for all $\zeta_{1}$ and $\zeta_{2}$ on $C$, where $\Delta s$ is the length of the (shorter) arc of $C$ between $\zeta_{1}$ and $\zeta_{2}$, then (5) and (6) hold uniformly for all $z_{0}=e^{i \theta_{0}}$ on the unit circle.

Proof. Let $k_{\rho}$ denote the arc of the circle $\left|z-z_{0}\right|=\rho<1$ which lies in $|z| \leqq 1$. We take $\rho \leqq a$ where $a$ is so small that the image of the arc $\left\{z|| z|=1| z-,z_{0} \mid \leqq a\right\}$ is a subarc of $\gamma$. Let $\Delta_{\rho}$ $=\left\{z|| z-z_{0}|<\rho| z \mid,<1\right\}, 0<\rho \leqq a$. We have

$$
l_{\rho}^{2}=\left(\int_{k_{\rho}}\left|f^{\prime}\left(z_{0}+\rho e^{i \phi}\right)\right| \rho d \phi\right)^{2} \leqq \int_{k_{\rho}}\left|f^{\prime}(z)\right|^{2} \rho d \phi \cdot \pi \rho\left(z=z_{0}+\rho e^{i \phi}\right),
$$

${ }^{2}$ Even the proofs given for the special case of Kellogg's theorem by Kellogg in [2] in 1912, by this author in [8] in 1932 and by Goluzin [1] in 1952 seem to be less elementary than the one given below. It may well be presented in an introductory course on conformal mapping.

Our proof of Theorem 1 still holds if the continuity of $\tau(s)$ for $\left|s-s_{0}\right| \leqq \sigma$ is replaced by the weaker assumption that $C$ has an " $L$-tangent" at $\zeta_{0}[5$, p. 93] and that $\left|\tau(s)-\tau\left(s_{0}\right)\right| \leqq \omega\left(\left|s-s_{0}\right|\right)$ for almost all $s$ in $\left|s-s_{0}\right| \leqq \sigma$. 
and therefore, for $0<r \leqq a$,

$$
\int_{0}^{r} \frac{l_{\rho}^{2}}{\rho} d \rho \leqq \pi \int_{0}^{r} \int_{k_{\rho}}\left|f^{\prime}(z)\right|^{2} \rho d \phi d \rho=\pi A(r)
$$

where $A(r)$ denotes the area of $f\left(\Delta_{r}\right)$. Because of (4) the length of boundary arc of $f\left(\Delta_{r}\right)$ which is a part of $C$ does not exceed $c l_{r}$ and therefore we have by the isoperimetric inequality

$$
A(r) \leqq \frac{\left(l_{r}+c l_{r}\right)^{2}}{4 \pi}=l_{r}^{2} \frac{(1+c)^{2}}{4 \pi} .
$$

Hence,

$$
F(r)=\int_{0}^{r} \frac{l_{\rho}^{2}}{\rho} d \rho \leqq l_{r}^{2}\left(\frac{1+c}{2}\right)^{2} .
$$

Since for almost all $r, 0<r \leqq a, r F^{\prime}(r)=l_{r}^{2}$ we have

$$
F(r) \leqq \frac{r}{2 \alpha} F^{\prime}(r), \quad \alpha=\frac{2}{(1+c)^{2}},
$$

or

$$
\frac{2 \alpha}{r} \leqq \frac{F^{\prime}(r)}{F(r)}
$$

and hence, for $0<r \leqq a$

$$
\frac{F(r)}{r^{2 \alpha}} \leqq \frac{F(a)}{a^{2 \alpha}} \leqq \frac{\pi A}{a^{2 \alpha}}=B_{0}
$$

where $A$ is the area of $\Omega$. Now there exists for every $r \leqq a$ an $r_{1}$ with $r / 2 \leqq r_{1} \leqq r$ such that

$$
l_{r_{1}}^{2} \log 2 \leqq F(r) \leqq B_{0 r}{ }^{2 \alpha}
$$

or

$$
l_{r_{1}} \leqq B r^{\alpha} .
$$

Thus, if $z$ is a point in $|z| \leqq 1$ with $\left|z-z_{0}\right|=r / 2$,

$$
\left|f(z)-f\left(z_{0}\right)\right| \leqq l_{r_{1}}(1+c) \leqq(1+c) B 2^{\alpha} \cdot\left(\frac{r}{2}\right)^{\alpha}=H\left|z-z_{0}\right|^{\alpha} .
$$

This proves (5). The inequality (6) follows from (5) by use of (4). 
In order to verify the corollary we only need to note that we can choose the constant $a$ such that, uniformly for all $z_{0}$ on $\left|z_{0}\right|=1$, the image of the arc $\left\{z|| z-z_{0}|\leqq a| z \mid,=1\right\}$ by means of $f(z)$ is shorter than one half of the length of $C$. Then the estimates (5) and (6) just derived hold uniformly for $\left|z_{0}\right|=1$.

Lemмa 2. Suppose $C$ is a closed rectifiable Jordan curve which possesses a continuous tangent in a neighborhood of a point $\zeta_{0}$. Let $\zeta=g(z)$ map the half-plane $\operatorname{Im}\{z\}>0$ conformally onto the interior of $C$ such that $g(0)=\zeta_{0}$. Then, uniformly for $0 \leqq \phi_{1} \leqq \phi_{2} \leqq \pi$,

$$
\lim _{\rho \rightarrow 0}\left|\frac{g\left(\rho e^{i \phi_{2}}\right)}{g\left(\rho e^{i \phi_{1}}\right)}\right|=1 .^{3}
$$

Proof. We may assume without loss of generality that the tangent to $C$ at $\zeta_{0}$ is the real and that the interior normal is the positive imaginary axis. By a theorem of Lindelöf [3] (see also [4, pp. 126-127; 1, pp. 371-372]) arg $g^{\prime}(z)^{4}$ has continuous boundary values on $y=0$ in a neighborhood of $z=0$, and

$$
\arg \left(\frac{g(z)}{z}\right) \rightarrow 0 \text { and } \arg g^{\prime}(z) \rightarrow 0
$$

as $z \rightarrow 0$ in $y \geqq 0$. For all sufficiently small $\rho$, say $0 \leqq \rho \leqq \rho_{0}$, the curve $\gamma_{\rho}: \zeta=g\left(\rho e^{i \phi}\right), 0 \leqq \phi \leqq \pi$ ( $\rho$ fixed), has, therefore, continuous tangent. Furthermore, if $\alpha$ denotes the tangent angle and $\psi$ the polar angle at the point $\zeta=g\left(\rho e^{i \phi}\right)$ we have

$$
\alpha-\psi=\arg g^{\prime}\left(\rho e^{i \phi}\right)+\phi+\frac{\pi}{2}-\arg g\left(\rho e^{i \phi}\right),
$$

and by (8), uniformly for $0 \leqq \phi \leqq \pi$,

$$
\left|\alpha-\psi-\frac{\pi}{2}\right| \leqq \delta(\rho), \quad \lim _{\rho \rightarrow 0} \delta(\rho)=0 .
$$

This inequality implies that any ray arg $\zeta=\psi$ intersects $\gamma_{\rho}$ in at most one point. Hence, $\boldsymbol{\gamma}_{\rho}$ may be represented in polar coordinates in the form $r=r(\psi)$, where $r^{\prime}(\psi)$ is continuous and

8 The conclusion of this lemma holds under a much weaker assumption on $C$ than that of the continuity of the tangent at $\zeta_{0}$ (e.g. if $C$ has a tangent at $\zeta_{0}$ and is "regulär unbewallt" as defined in [7, p. 357]; see J. Wolff [9]). The above formulation may also be obtained from a result of Ostrowski $[5$, p. 118], but for the sake of completeness we give an independent proof.

Since $g^{\prime}(z) \neq 0$ in $y>0$, arg $f^{\prime}(z)$ is single-valued once it is normalized at one point. 


$$
\left|\frac{r^{\prime}(\psi)}{r(\psi)}\right|=|\cot (\alpha-\psi)| \leqq \tan \delta(\rho) .
$$

By the mean value theorem we have, therefore, for any $\psi_{1}$ and $\psi_{2}$ in the interval in which $r(\psi)$ is defined,

$$
\left|\log r\left(\psi_{2}\right)-\log r\left(\psi_{1}\right)\right| \leqq\left|\psi_{2}-\psi_{1}\right| \tan \delta(\rho) \leqq 2 \pi \tan \delta(\rho),
$$

since $\left|\psi_{2}-\psi_{1}\right| \leqq 2 \pi$. This inequality implies the conclusion (7).

2. Proof of Theorem 1. Since $C$ has a continuous tangent in a neighborhood of $\zeta_{0}$, it follows from Lindelöf's theorem [3] that $\arg f^{\prime}(z)$ assumes continuous boundary values on an $\operatorname{arc} \beta$ of $|z|=1$ which contains $z_{0}$ as interior point. We normalize $\arg f^{\prime}(z)$ by requiring that $\arg f^{\prime}(0)=0$. The rectifiability of $C$ insures that $\arg f^{\prime}(z)$ has boundary values, which we denote by $\arg f^{\prime}\left(e^{i \theta}\right)$, almost everywhere on $|z|=1$. Without loss of generality we may make the further assumption that $\arg f^{\prime}(z)$ may be represented by the Poisson integral in $|z|<1$ with the boundary function $\arg f^{\prime}\left(e^{i \theta}\right)$. Otherwise, we construct a closed Jordan curve $\Gamma$ with continuous tangent, which has a subarc $\beta^{\prime}$ of $\beta$, containing $z_{0}$ as interior point, in common with $|z|=1$ and is otherwise contained in $|z|<1$. We may also assume that $z=0$ is in the interior of $\Gamma$. Let $z=h(w)$ map the disk $|w|<1$ conformally onto the interior of $\Gamma, h(0)=0$. Then $\arg \{(d / d w) f[h(w)]\}=\arg f^{\prime}[h(w)]$ $+\arg h^{\prime}(w)$ is continuous in $|w| \leqq 1$ and is thus representable by the Poisson integral in $|w|<1$. Since $h^{-1}(z)$ is analytic on $\beta^{\prime}$ with a nonvanishing derivative at $z_{0}$, it is sufficient to prove the theorem for $f[h(w)]$ in order to insure its validity for $f(z)$.

Under the assumption that $\arg f^{\prime}(z)$ is represented by the Poisson integral in $|z|<1$ the conjugate function $\log \left|f^{\prime}(z)\right|$ approaches

(9) $h\left(\theta_{0}\right)=\frac{1}{2 \pi} \int_{0}^{\pi}\left[\arg f^{\prime}\left(z_{0} e^{i t}\right)-\arg f^{\prime}\left(z_{0} e^{-i t}\right)\right] \cot \frac{t}{2} d t+\log \left|f^{\prime}(0)\right|$

as $z \rightarrow z_{0}=e^{i \theta_{0}}$ in any Stolz angle, if the latter integral converges absolutely $\left[10\right.$, p. 54]. This together with the continuity of $\arg f^{\prime}(z)$ at $z_{0}$ will establish (2), with $f^{\prime}\left(z_{0}\right)=\exp \left[h\left(\theta_{0}\right)+i \arg f^{\prime}\left(e^{i \theta_{0}}\right)\right]$.

To prove the absolute convergence of (9) we note that

$$
\arg f^{\prime}\left(e^{i \theta}\right)=\tau[s(\theta)]-\theta-\frac{\pi}{2}
$$

and therefore

$$
\begin{aligned}
\left|\arg f^{\prime}\left(e^{i\left(\theta_{0}+t\right)}\right)-\arg f^{\prime}\left(e^{i \theta_{0}}\right)\right| & \leqq\left|\tau\left[s\left(\theta_{0}+t\right)\right]-\tau\left[s\left(\theta_{0}\right)\right]\right|+|t| \\
& \leqq \omega\left(K|t|^{\alpha}\right)+|t|
\end{aligned}
$$


by (6). Hence, for some $\delta, 0<\delta<\pi$,

$$
\text { (10) } \begin{aligned}
\int_{0}^{\delta}\left|\arg f^{\prime}\left(e^{i\left(\theta_{0}+t\right)}\right)-\arg f^{\prime}\left(e^{i \theta_{0}}\right)\right| & \cot \frac{t}{2} d t \leqq 2 \int_{0}^{\delta} \frac{\omega\left(K t^{\alpha}\right)}{t} d t+2 \delta \\
& =\frac{2}{\alpha} \int_{0}^{K \delta^{\alpha}} \frac{\omega(u)}{u} d u+2 \delta .
\end{aligned}
$$

Finally, to prove (1) we note first that the relation (2) implies (1) in any Stolz angle. To obtain (1) also for unrestricted approach we apply Lemma 2 . Let $c_{\rho}$ denote the circle of radius $\rho(0<\rho<1)$ orthogonal to the circle $|z|=1$ which contains $z_{0}$ in its interior and intersects $|z|=1$ at points equidistant from $z_{0}$. Then, for any $z_{1}$ and $z_{2}$ on $c_{\rho}$, by Lemma 2,

$$
\lim _{\rho \rightarrow 0}\left|\frac{f\left(z_{2}\right)-f\left(z_{0}\right)}{f\left(z_{1}\right)-f\left(z_{0}\right)}\right|=1,
$$

uniformly in $z_{1}, z_{2}$. Thus, if $z_{1}$ is chosen on the radius ending at $z_{0}$ and $z=z_{2}$ is an arbitrary point on $c_{\rho}$,

$$
\left|\frac{f(z)-f\left(z_{0}\right)}{z-z_{0}}\right| \sim\left|\frac{f\left(z_{1}\right)-f\left(z_{0}\right)}{z_{1}-z_{0}}\right| \quad \text { as } \rho \rightarrow 0 .
$$

But since the second term has the limit $\left|f^{\prime}\left(z_{0}\right)\right|=\exp \left[h\left(\theta_{0}\right)\right]$ it follows that

$$
\lim _{z \rightarrow z_{0}}\left|\frac{f(z)-f\left(z_{0}\right)}{z-z_{0}}\right|=\left|f^{\prime}\left(z_{0}\right)\right|
$$

for unrestricted approach in $|z| \leqq 1$. Moreover, by Lindelöf [3]

$$
\lim _{z \rightarrow z_{0}}\left[\arg \frac{f(z)-f\left(z_{0}\right)}{z-z_{0}}\right]=\arg f^{\prime}\left(z_{0}\right)
$$

for unrestricted approach. Hence (11) and (12) imply (1).

3. Proof of Theorem 2. The assumption that the $C$ possesses a continuous tangent throughout assures (1) that the corollary to Lemma 1 holds and (2) that arg $f^{\prime}(z)$ has continuous boundary values on $|z|=1$. Then the argument of the preceding section proving the absolute convergence of the integral (9), shows that it converges uniformly for all $\theta_{0}$ in $[0,2 \pi]$. Hence $f^{\prime}(z)$ is continuous in $|z| \leqq 1$.

The statement regarding the modulus of continuity of $\arg f^{\prime}\left(e^{i \theta}\right)$ follows immediately from the inequalities $(t>0)$ 


$$
\begin{aligned}
\left|\arg f^{\prime}\left(e^{i\left(\theta_{0}+t\right)}\right)-\arg f^{\prime}\left(e^{i \theta_{0}}\right)\right| & \leqq\left|\tau\left[s\left(\theta_{0}+t\right)\right]-\tau\left[s\left(\theta_{0}\right)\right]\right|+t \\
& \leqq \omega(m t)+t=\omega_{0}(t) .
\end{aligned}
$$

Then the expression given for $\omega_{0}^{*}(\theta)$ follows from a theorem of J. L. Walsh and Margaret Elliott [6].

\section{REFERENCES}

1. G. M. Groluzin, Geometrische Funktionentheorie, Berlin, Deutscher Verlag der Wissenschaften, 1957 (Transl. of 1952 Russian edition).

2. O. D. Kellogg, Harmonic functions and Green's integral, Trans. Amer. Math. Soc. vol. 13 (1912) pp. 109-132.

3. E. Lindelöf, Sur la représentation conforme d'une aire simplement connexe sur l'aire d'un cercle, C. R. du Quatrième Congrès des Math. Scand. à Stockholm (1916) pp. 59-90. 1944.

4. J. E. Littlewood, Lectures on the theory of functions, Oxford University Press,

5. A. Ostrowski, Über den Habitus der konformen Abbildung am Rande des $A b$ bildungsbereiches, Acta Math. vol. 64 (1935) pp. 81-185.

6. J. L. Walsh and Margaret Elliott, Degree of approximation on a Jordan curve, Proc. Nat. Acad. Sci. U.S.A. vol. 38 (1952) pp. 1058-1066.

7. S. E. Warschawski, Über das Randverhalten der Ableitung der Abbildungsfunktion bei konformer Abbildung, Math. Z. vol. 35 (1932) pp. 321-456.

8. - Über einen Satz von O. D. Kellogg, Göttinger Nachrichten, Math.-Phys. Klasse, 1932, pp. 73-86.

9. J. Wolff, Sur la représentation conforme des bandes, Compositio Math. vol. 1 (1934) pp. 217-222.

10. A. Zygmund, Trigonometrical series, Warsaw, 1935.

UNIVERSITY OF MiNNESOTA 